Macedonian Pharmaceutical Bulletin, 66 (Suppl 1) 113 - 114 (2020)

Online ISSN 1857 - 8969

UDC: $615.015: 620.3$

DOI: 10.33320/maced.pharm.bull.2020.66.03.056

Short communication

\title{
The use of dendrimers as a modern drug delivery platform
}

\author{
Elena Drakalska Sersemova ${ }^{1}$, Tanja Vasileva ${ }^{2}$, Bistra Angelovska $^{1}$, Dijana Miceva ${ }^{1}$, \\ Natasha Miteva ${ }^{1}$ \\ ${ }^{1}$ Faculty of Medical Sciences, University “Goce Delcev”- Stip, Krste Misirkov 10-A, 2000 Stip, N. Macedonia \\ ${ }^{2}$ Oaza Alkaloidi, Gladno Pole, Village Tarinci, Municipality Karbinci 2207, N. Macedonia
}

\section{Introduction}

The therapeutic potential of many active substances cannot be realized in clinical practice due to adverse physic-chemical properties, variable pharmacokinetics and a range of adverse effects causing low bioavailability and unsatisfactory therapeutic concentration in the target tissue. In order to overcome these problems, in recent years the emphasis has been placed on the study and characterization of different types of nanoparticles that offer many advantages over conventional therapy. The most common investigated and characterized nanosystems are dendrimers, as a new class of polymers.

Dendrimers as nanosystems are formulated from materials that are biocompatible, biodegradable and non-immunogenic. They have unique properties like thermodynamic stability, small particle size (1-20 $\mathrm{nm}$ ), low polydispersity index and negative zeta potential (Svanson et al., 2018). The dendrimer structure is compatible with a range of hydrophilic and hydrophobic active substances with high encapsulation efficiency using different preparation methods (Abasi et al., 2014).

There are a several types of used dendrimers but PAMAM dendrimers are the most commonly studied dendrimers due to documented safety studies, and are candidates for encapsulating a large number of antineoplastic agents (Dubey et al., 2019).
The therapeutic efficacy of many antineoplastics is limited due to their poor penetration into tumor tissue and serious adverse effects on healthy cells. This section discusses the results of clinical trials incorporating the most commonly used antineoplastic drugs in dendrimer formulations (Mariyam et al., 2018).

\section{Materials and methods}

The propose of this paper is to review the structure of the dendrimers and the role of the components in their structure as well as compare and detect the factors which affect the stability of dendrimers and developing different formulation methods. We did a research for characterization of dendrimers with encapsulated active substance and processing and comparison of clinical trial results on the efficacy and toxicity of drugs incorporated into dendrimers against different types of disease.

Our research is made for characterization of incorporating the most commonly used antineoplastics drugs (doxorubicin, paclitaxel and docetaxel, camptothecin) in dendrimer formulations.

To accomplish these goals, we used data from relevant literature sources from primary, secondary and tertiary literature, with emphasis on original scientific research on the characterization and evaluation of dendrimers with incorporated active substances and processing of their results. We systematized the summarized literary data according

\footnotetext{
* elena.drakalska@ugd.edu.mk
} 
to the actual treatment of the problem, noted the formulation aspects of using different methods and active substances, and identified the advantages and disadvantages by highlighting the possibilities of optimizing the therapy of various diseases of the dendrimer. We discussed the processed results, tabulated the patented products, and drew appropriate conclusions from the evaluated results.

\section{Results and discussion}

Doxorubicin is used in the treatment of several types of cancers, but due to the adverse effects, several formulations of doxorubicin with dendrimers have been investigated, and the results have shown that $\mathrm{P} 1$ and $\mathrm{P} 2$ particles inhibit cancer growth twice as much, and that is result of the additive effect of complex of dendrimers with DOX, as well as a significant reduction in side effects (Zhang et al., 2018).

PAMAM dendrimers have been proven to be ideal platforms for delivering also paclitaxel and docetaxel, significantly increasing water solubility and providing higher bioavailability (Li et al., 2018). Encapsulation of camptothecin resulted in a significantly higher percentage of accumulation in malignant cells compared to the free drug (Thiagarajan et al., 2010).

In addition to the widespread use of antineoplastic delivery, numerous clinical studies have shown that dendrimers, especially third- and fifth-generation PAMAM, are promising candidates for transdermal, oral and ocular delivery of numerous active substances with adverse pharmacokinetic properties. It is possible to modify the membrane with specific ligands that will provide targeted delivery to certain tumor cells with overexpressing receptors that will allow controlled release of the active substance into the malignant cell without affecting the surrounding tissues (Wolinsky et al., 2018).

\section{Conclusion}

Dendrimers are a challenge in modern therapy given the fact that they provide targeted delivery of drugs that can increase the bioavailability of the encapsulated drug and enable controlled and prolonged therapeutic activity.
The high cost, low solubility, volatility at certain $\mathrm{pH}$ values and short half-life make the application of other nanoparticles significantly difficult. Dendrimers provide controlled delivery of different types of active substances followed by increased therapeutic effect, achieving high concentration in the target cell and reducing the adverse effects

From the processed data, we concluded that dendrimers are optimal carriers for many active substances providing higher solubility, greater stability and improved bioavailability, especially at antineoplastic treatment.

\section{References}

Abbasi, E., Aval, S.F., Akbarzadeh, A., Milani, M., Nasrabadi, H.T., Joo, S.W., Hanifehpour, Y., NejatiKoshki, K., Pashaei-Asl, R., 2014. Dendrimers: synthesis, applications, and properties. Nanoscale research letters 9(1), 247.

Dubey, S., Salunkhe, S., Agrawal, M., Kali, M., Singhvi, G., Tiwari, S., Saraf, S., Shailendra, S., Alexander, A., 2019. Understanding the pharmaceutical aspects of dendrimers for the delivery of anticancer drugs. Current Drug Targets.

Li, X., Sun, A., Liu, Y., Zhang, W., Pang, N., Cheng, S., Qi, X., 2018. Amphiphilic dendrimer engineered nanocarrier systems for co-delivery of siRNA and paclitaxel to matrix metalloproteinase-rich tumors for synergistic therapy. NPG Asia Mater 10, 238-254.

Mariyam, M., Ghosal, K., Thomas, S., Nandakumar, K., Latha, M., 2018. Dendrimers: general Aspects, Applications and Structural exploitations as prodrug/drug-delivery vehicles in current medicine. Mini-Reviews in Medicinal Chemistry 18, 439.

Thiagarajan, G., Ray, A., Malugin, A., Ghandehari, H., 2010. PAMAM-camptothecin conjugate inhibits proliferation and induces nuclear fragmentation in colorectal carcinoma cells. Pharmaceutical research 27(11), 2307-2316.

Wolinsky, J. and Grinstaff, M., 2018. Therapeutic and diagnostic applications of dendrimers for cancer treatment. Advanced Drug Delivery Reviews 60(9), $1037-1055$.

Zhang, M., Zhu, J., Zheng, Y., Guo, R., Wang, S., Mignani, S., Caminade A.M., Majora, J.P., Shi, X., 2018. Doxorubicin-conjugated PAMAM dendrimers for pH-responsive drug Release and Folic Acidtargeted cancer therapy. Pharmaceutics 10(3), 162. 